\title{
CUSPIDAL MODULAR SYMBOLS ARE TRANSPORTABLE
}

\author{
WILLIAM A. STEIN AND HELENA A. VERRILL
}

\begin{abstract}
Modular symbols of weight 2 for a congruence subgroup $\Gamma$ satisfy the identity $\{\alpha, \gamma(\alpha)\}=\{\beta, \gamma(\beta)\}$ for all $\alpha, \beta$ in the extended upper half plane and $\gamma \in \Gamma$. The analogue of this identity is false for modular symbols of weight greater than 2 . This paper provides a definition of transportable modular symbols, which are symbols for which an analogue of the above identity holds, and proves that every cuspidal symbol can be written as a transportable symbol. As a corollary, an algorithm is obtained for computing periods of cuspforms.
\end{abstract}

\section{Introduction}

It is well known that modular symbols of weight 2 for a congruence subgroup $\Gamma$ satisfy the identity $\{\alpha, \gamma(\alpha)\}=\{\beta, \gamma(\beta)\}$ for all $\alpha, \beta$ in the extended upper half plane and $\gamma \in \Gamma$. The analogue of this identity is, in general, false for modular symbols of weight greater than 2. To investigate further, we define transportable modular symbols, which are symbols that can be expressed in such a way that an analogue of the above identity holds. We then prove that every cuspidal symbol is transportable. As a corollary, we obtain an algorithm for computing periods of cuspforms.

In Section 1 we review the definition of modular symbols. In Section 2 we define transportable modular symbols, and prove our main theorem. Section 3 contains an application of our transportability result to the computation of periods of modular forms. Finally, Section 4 contains two examples in which we verify the assertion of Theorem 2.4 and apply the period computation algorithm.

\section{Modular symbols}

In Section 1.1 we recall the definition of modular symbols given in [5]; then in Section 1.2 we introduce a slight generalization of the definition. Let $N$ and $k$ be positive integers with $k \geqslant 2$, and let $\varepsilon: \mathbb{Z} / N \mathbb{Z} \rightarrow \mathbb{C}$ be a Dirichlet character modulo $N$.

\subsection{Definition}

Let $\mathcal{M}$ be the abelian group generated by all symbols $\{\alpha, \beta\}$ with $\alpha, \beta \in \mathbb{P}^{1}(\mathbb{Q})$, modulo the relations $\{\alpha, \beta\}+\{\beta, \gamma\}+\{\gamma, \alpha\}=0$, and modulo any torsion. Let $V_{k-2}$ denote the group of homogeneous polynomials in $\mathbb{Z}[X, Y]$ of degree $k-2$. 
Each element $\gamma=\left(\begin{array}{ll}a & b \\ c & d\end{array}\right) \in \mathrm{SL}(2, \mathbb{Z})$ acts on the left on $V_{k-2}$ by

$$
\gamma(P(X, Y))=P(d X-b Y,-c X+a Y),
$$

and on $\mathcal{M}_{k}=V_{k-2} \otimes \mathcal{M}$ by

$$
\gamma(P \otimes\{\alpha, \beta\})=\gamma(P) \otimes\{\gamma(\alpha), \gamma(\beta)\} .
$$

Fix a Dirichlet character $\varepsilon: \mathbb{Z} / N \mathbb{Z} \rightarrow \mathbb{C}$, and denote by $\mathbb{Z}[\varepsilon]$ the ring generated by the image of $\varepsilon$. We also view $\varepsilon$ as a homomorphism $\Gamma_{0}(N) \rightarrow \mathbb{C}^{*}$ by setting $\varepsilon\left(\begin{array}{ll}a & b \\ c & d\end{array}\right)=\varepsilon(d)$.

The space $\mathcal{M}_{k}(N, \varepsilon)$ of modular symbols of level $N$ and character $\varepsilon$ is the quotient of the $\mathbb{Z}[\varepsilon]$-module $\mathcal{M}_{k} \otimes \mathbb{Z}[\varepsilon]$ by the $\mathbb{Z}[\varepsilon]$-submodule generated by $\gamma(x)-\varepsilon(\gamma) x$ for all $x \in \mathcal{M}_{k}$, for all $\gamma \in \Gamma_{0}(N)$, and by any torsion. Denote by $P\{\alpha, \beta\}$ the image of $P \otimes\{\alpha, \beta\}$ in $\mathcal{M}_{k}(N, \varepsilon)$. The $\mathbb{Q}[\varepsilon]$-vector space

$$
\mathcal{M}_{k}(N, \varepsilon ; \mathbb{Q})=\mathcal{M}_{k}(N, \varepsilon) \otimes_{\mathbb{Z}} \mathbb{Q}
$$

contains $\mathcal{M}_{k}(N, \varepsilon)$.

Let $\mathcal{B}$ be the free abelian group generated by all symbols $\{\alpha\}$, for $\alpha \in \mathbb{P}^{1}(\mathbb{Q})$. Define a left action of $\operatorname{SL}(2, \mathbb{Z})$ on $\mathscr{B}_{k}=V_{k-2} \otimes \mathscr{B}$ by

$$
\gamma(P \otimes\{\alpha\})=\gamma(P) \otimes\{\gamma \alpha\} .
$$

The space $\mathcal{B}_{k}(N, \varepsilon)$ of boundary symbols is the quotient of $\mathscr{B}_{k} \otimes \mathbb{Z}[\varepsilon]$ by the $\mathbb{Z}[\varepsilon]$-submodule generated by $\gamma(x)-\varepsilon(\gamma) x$ for all $x \in \mathscr{B}_{k}$, for all $\gamma \in \Gamma_{0}(N)$, and by any torsion. The subspace $\oiint_{k}(N, \varepsilon)$ of cuspidal symbols is the kernel of the map $\delta: \mathcal{M}_{k}(N, \varepsilon) \rightarrow \mathscr{B}_{k}(N, \varepsilon)$ given by $\delta(P\{\alpha, \beta\})=P\{\beta\}-P\{\alpha\}$.

When $\varepsilon=1$ is the trivial character, we shall also write $\mathcal{M}_{k}\left(\Gamma_{0}(N)\right)$ for $\mathcal{M}_{k}(N, 1)$, and similarly for $\diamond_{k}$ and $\mathscr{B}_{k}$.

\subsection{Extended modular symbols}

It is useful to extend the notion of modular symbols to allow symbols of the form $P\{z, w\}$ where $z$ and $w$ are arbitrary elements of $\mathfrak{h}^{*}=\mathfrak{h} \cup \mathbb{P}^{1}(\mathbb{Q})$.

Definition 1 (Extended modular symbols). The group $\overline{\mathcal{M}}_{k}$ of extended modular symbols is the free abelian group with basis the set of all symbols $P\{z, w\}$ with $z, w \in \mathfrak{h}^{*}$, subject to the relations $P\{u, v\}+P\{v, w\}+P\{w, u\}=0$.

Note that $\overline{\mathcal{M}}_{k}$ is of uncountable rank over $\mathbb{Z}$. It is equipped with an action of $\Gamma_{0}(N)$; we let $\overline{\mathcal{M}}_{k}(N, \varepsilon)$ be the largest torsion-free quotient of $\overline{\mathcal{M}}_{k}$ by the relations $\gamma x=\varepsilon(\gamma) x$ for $\gamma \in \Gamma_{0}(N)$.

\section{Transportable modular symbols}

In Section 2.1 we define transportable modular symbols, and we prove an elementary proposition that motivates the definition. Section 2.2, which is the heart of this paper, contains a proof that every cuspidal modular symbol is transportable.

\subsection{Definition}

Definition 2 (Transportable). A modular symbol is transportable if it can be written in the form

$$
\sum_{i=1}^{m} P_{i}\left\{\infty, \gamma_{i}(\infty)\right\}
$$


for $\gamma_{i} \in \Gamma_{0}(N)$ and $P_{i} \in V_{k-2}$ with

$$
\sum_{i=1}^{m} P_{i}\left\{\infty, \gamma_{i}(\infty)\right\}=\sum_{i=1}^{m} P_{i}\left\{\alpha, \gamma_{i}(\alpha)\right\}
$$

for all $\alpha \in \mathfrak{h}^{*}$, where the equality takes place in $\overline{\mathcal{M}}_{k}(N, \varepsilon)$.

When $k=2$, the identity $\{\infty, \gamma(\infty)\}=\{\alpha, \gamma(\alpha)\}$ holds for any $\alpha \in \mathfrak{h}^{*}$, so in weight 2 there is a plentiful supply of transportable modular symbols.

Proposition 2.1. For any $\gamma \in \Gamma_{0}(N), P \in V_{k-2}$ and $\alpha \in \mathfrak{h}^{*}$,

$$
\begin{aligned}
P\{\infty, \gamma(\infty)\} & =P\{\alpha, \gamma(\alpha)\}+\left(P-\varepsilon(\gamma) \gamma^{-1} P\right)\{\infty, \alpha\} \\
& =\varepsilon(\gamma)\left(\gamma^{-1} P\right)\{\alpha, \infty\}-P\{\gamma(\alpha), \infty\} .
\end{aligned}
$$

In particular,

$$
P\{\infty, \gamma(\infty)\}=P\{\alpha, \gamma(\alpha)\} \Leftrightarrow P=\varepsilon(\gamma) \gamma^{-1} P \text {. }
$$

Proof. If $x \in \mathcal{M}_{k}(N, \varepsilon)$ is a modular symbol and $\gamma \in \Gamma_{0}(N)$, then $\gamma x=\varepsilon(\gamma) x$, where, as usual, $\varepsilon$ is viewed as a homomorphism $\Gamma_{0}(N) \rightarrow \mathbb{C}^{*}$ via $\varepsilon\left(\left(\begin{array}{ll}a & b \\ c & d\end{array}\right)\right)=\varepsilon(d)$. In particular, $\varepsilon(\gamma) \gamma^{-1} x=x$, so

$$
\begin{aligned}
P\{\infty, \gamma(\infty)\} & =P\{\infty, \alpha\}+P\{\alpha, \gamma(\alpha)\}+P\{\gamma(\alpha), \gamma(\infty)\} \\
& =P\{\infty, \alpha\}+P\{\alpha, \gamma(\alpha)\}+\varepsilon(\gamma) \gamma^{-1}(P\{\gamma(\alpha), \gamma(\infty)\}) \\
& =P\{\infty, \alpha\}+P\{\alpha, \gamma(\alpha)\}+\varepsilon(\gamma)\left(\gamma^{-1} P\right)\{\alpha, \infty\} \\
& =P\{\alpha, \gamma(\alpha)\}+P\{\infty, \alpha\}-\varepsilon(\gamma)\left(\gamma^{-1} P\right)\{\infty, \alpha\} \\
& =P\{\alpha, \gamma(\alpha)\}+\left(P-\varepsilon(\gamma) \gamma^{-1} P\right)\{\infty, \alpha\} .
\end{aligned}
$$

The remaining statements of the proposition now follow easily.

Example 2.2. In some cases it is easy to give a formula for symbols that are obviously transportable. Suppose that $k \geqslant 2$ is an even integer. If $P$ is a polynomial such that $\gamma(P)=P$ for some $\gamma \in \Gamma_{0}(N)$, then $P\{\infty, \gamma(\infty)\}$ is transportable. Given $\gamma \in \Gamma_{0}(N)$, an example of such a $P$ is

$$
P(X, Y)=\left(c X^{2}+(d-a) X Y-b Y^{2}\right)^{(k-2) / 2} .
$$

We found this polynomial by viewing $V_{k-2}$ as the $(k-2)$ th symmetric product of the 2-dimensional space on which $\Gamma_{0}(N)$ acts naturally. If $\gamma$, which has determinant 1 , has eigenvalues $\alpha$ and $\alpha^{-1}$, then the eigenvalues of the $(k-2)$-fold symmetric product of $\gamma$ are given by $\alpha^{k-2-2 j}$ for $0 \leqslant j \leqslant k-2$. Although we have not been able to find a counterexample, the authors see no reason to believe that transportable symbols of the form given in this example always span $\wp_{k}(N ; \mathbb{Q})$.

More generally, given any sequence of matrices $\gamma_{1}, \ldots, \gamma_{n}$ in $\Gamma_{0}(N)$, it is a simple matter of linear algebra to give transportable symbols of the form $\sum_{i=1}^{n} P_{i}\left\{\infty, \gamma_{i} \infty\right\}$. This follows from Lemma 2.3, which shows that this symbol is transportable exactly when $\left(P_{1}, \ldots, P_{n}\right)$ is in the kernel of the map $\bigoplus_{i=1}^{n}\left(1-\gamma_{i}^{-1}\right)$ from $\bigoplus_{i=1}^{n} V_{k-2}$ to $V_{k-2}$. 


\subsection{Characterization of transportable modular symbols}

Lemma 2.3. A modular symbol in $\mathcal{M}_{k}(N, \varepsilon ; \mathbb{Q})$ is transportable if and only if it can be written in the form $\sum_{i=1}^{m} P_{i}\left\{\infty, \gamma_{i}(\infty)\right\}$ with

$$
\sum P_{i}=\sum \varepsilon\left(\gamma_{i}\right) \gamma_{i}^{-1} P_{i}
$$

Proof. This follows from Proposition 2.1.

Figure 1 illustrates Lemma 2.3 with a trivial-character example.

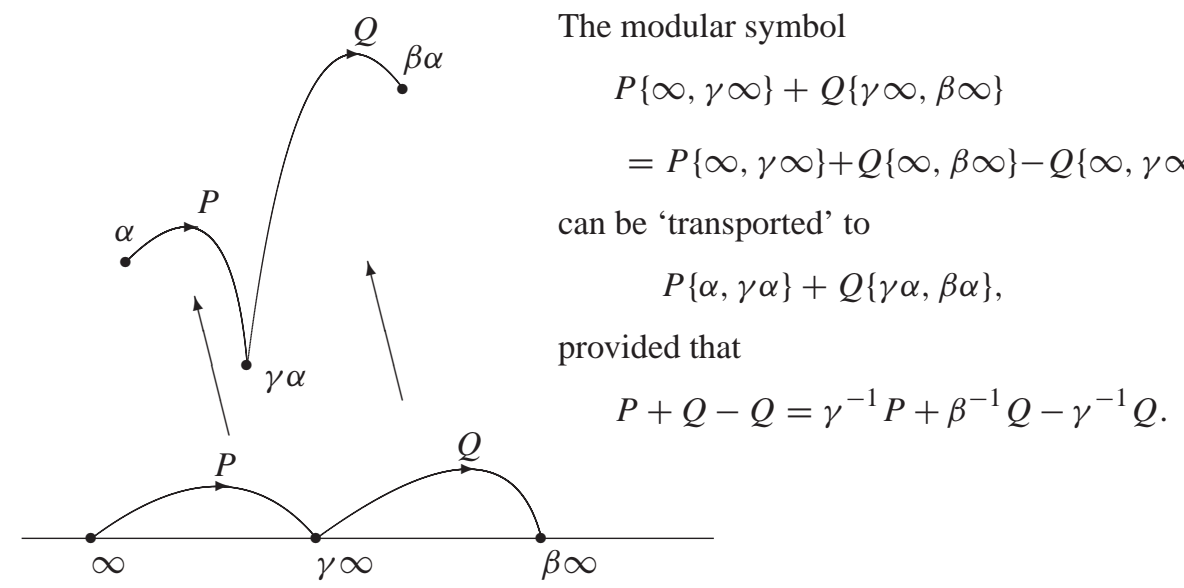

Figure 1: 'Transporting' a transportable modular symbol.

Theorem 2.4. A modular symbol is transportable if and only if it is cuspidal.

Proof. By Lemma 2.3, every transportable modular symbols is cuspidal, so we must prove that every cuspidal symbol is transportable.

Let $I=I_{N, \varepsilon}$ be the ideal in the group ring of $\Gamma_{0}(N)$ generated by all elements of the form $\varepsilon(\gamma)-\gamma$ for $\gamma \in \Gamma_{0}(N)$. Suppose that $v \in \oiint_{k}(N, \varepsilon)$. Use the relation $\{\alpha, \beta\}=\{\infty, \beta\}-\{\infty, \alpha\} \in \mathcal{M}$ to see that any $v$ is the image of an element $\tilde{v} \in \mathcal{M}_{k}$ of the form

$$
\tilde{v}=\sum_{\beta \in \mathbb{Q}} P_{\beta} \otimes\{\infty, \beta\} \in \mathcal{M}_{k}
$$

with only finitely many $P_{\beta}$ nonzero. For later convenience, we set $P_{\infty}=0$, and take sums over all $\beta \in P^{1}(\mathbb{Q})$. The boundary map $\delta$ lifts in a natural way to $\mathcal{M}_{k}=V_{k-2} \otimes \mathcal{M}$, as illustrated.

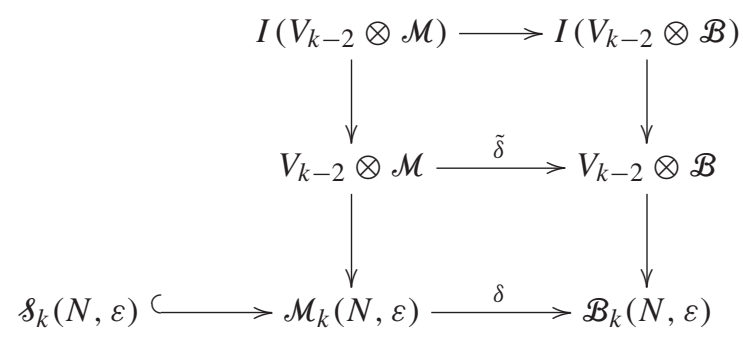


Bearing in mind torsion, our assumption that $\delta(v)=0$ implies that for some nonzero $M \in \mathbb{Z}$, we have $M \tilde{\delta}(\tilde{v}) \in I\left(V_{k-2} \otimes \mathcal{B}\right)$. So there are $Q_{\gamma, \beta} \in V_{k-2}$, for $\gamma \in \Gamma_{0}(N)$ and $\beta \in \mathbb{P}^{1}(\mathbb{Q})$, only finitely $\beta$ many nonzero, such that

$$
M \tilde{\delta}(\tilde{v})=\sum_{\gamma, \beta}(\varepsilon(\gamma)-\gamma)\left(Q_{\gamma, \beta} \otimes\{\beta\}\right) .
$$

We now use a summation trick.

$$
\begin{aligned}
M \tilde{\delta}(\tilde{v}) & =M \sum_{\beta}\left(P_{\beta} \otimes\{\beta\}-P_{\beta} \otimes\{\infty\}\right) \\
& =\sum_{\gamma, \beta}\left(\varepsilon(\gamma) Q_{\gamma, \beta} \otimes\{\beta\}-\left(\gamma Q_{\gamma, \beta}\right) \otimes\{\gamma \beta\}\right) \\
& =\sum_{\gamma, \beta} \varepsilon(\gamma) Q_{\gamma, \beta} \otimes\{\beta\}-\left(\gamma Q_{\gamma, \gamma^{-1} \beta}\right) \otimes\{\beta\} \\
& =\sum_{\gamma, \beta}\left(\varepsilon(\gamma) Q_{\gamma, \beta}-\gamma Q_{\gamma, \gamma^{-1} \beta}\right) \otimes\{\beta\} .
\end{aligned}
$$

This shows that

$$
M \sum_{\beta}\left(P_{\beta} \otimes\{\beta\}-P_{\beta} \otimes\{\infty\}\right)=\sum_{\gamma, \beta}\left(\varepsilon(\gamma) Q_{\gamma, \beta}-\gamma Q_{\gamma, \gamma^{-1} \beta}\right) \otimes\{\beta\} .
$$

Equating terms, we deduce that for $\beta \neq \infty$,

$$
M P_{\beta}=\sum_{\gamma}\left(\varepsilon(\gamma) Q_{\gamma, \beta}-\gamma Q_{\gamma, \gamma^{-1} \beta}\right)
$$

Using this expression for $P_{\beta}$, as well as the fact that $\varepsilon(\gamma) \gamma^{-1}$ acts trivially on $\mathcal{M}_{k}(N, \varepsilon)$, we find that

$$
\begin{aligned}
M v=M \sum_{\beta} P_{\beta}\{\infty, \beta\} & =\sum_{\gamma, \beta}\left(\varepsilon(\gamma) Q_{\gamma, \beta}-\gamma Q_{\gamma, \gamma^{-1} \beta}\right)\{\infty, \beta\} \\
& =\sum_{\gamma, \beta} \varepsilon(\gamma) Q_{\gamma, \beta}-\varepsilon(\gamma) \gamma^{-1}\left(\left(\gamma Q_{\gamma, \gamma^{-1} \beta}\right)\{\infty, \beta\}\right) \\
& =\sum_{\gamma, \beta} \varepsilon(\gamma) Q_{\gamma, \beta}\{\infty, \beta\}-\varepsilon(\gamma) Q_{\gamma, \gamma^{-1} \beta}\left\{\gamma^{-1} \infty, \gamma^{-1} \beta\right\} \\
& =\sum_{\gamma, \beta} \varepsilon(\gamma) Q_{\gamma, \beta}\{\infty, \beta\}-\varepsilon(\gamma) Q_{\gamma, \beta}\left\{\gamma^{-1} \infty, \beta\right\} \\
& =\sum_{\gamma, \beta} \varepsilon(\gamma) Q_{\gamma, \beta}\left\{\infty, \gamma^{-1} \infty\right\} .
\end{aligned}
$$

Equating coefficients of $\{\infty\}$ in Equation 3, we have

$$
-M \sum_{\beta} P_{\beta}=\sum_{\gamma}\left(\varepsilon(\gamma) Q_{\gamma, \infty}-\gamma Q_{\gamma, \gamma^{-1} \infty}\right),
$$

which, combining with Equation 4, and recalling that $P_{\infty}=0$, means that

$$
-\sum_{\gamma, \beta \neq \infty}\left(\varepsilon(\gamma) Q_{\gamma, \beta}-\gamma Q_{\gamma, \gamma^{-1} \beta}\right)=\sum_{\gamma}\left(\varepsilon(\gamma) Q_{\gamma, \infty}-\gamma Q_{\gamma, \gamma^{-1} \infty}\right),
$$


and hence

$$
\sum_{\gamma, \beta}\left(\varepsilon(\gamma) Q_{\gamma, \beta}-\gamma Q_{\gamma, \beta}\right)=0 .
$$

Using the expression

$$
v=-\frac{1}{M} \sum_{\beta, \gamma} \varepsilon(\gamma) Q_{\gamma, \beta}\left\{\infty, \gamma^{-1} \infty\right\}
$$

obtained from Equation 5, we see that this is the condition for $v$ to be transportable.

Corollary 2.5. Fix $\alpha \in \mathfrak{h}^{*}$. Every element of $\S_{k}(N, \varepsilon)$ is a sum of modular symbols of the form $P\{\alpha, \gamma(\alpha)\}$.

Proof. Let $x \in \wp_{k}(N, \varepsilon)$. Proposition 2.1 implies that $x$ is transportable, so there exist $P_{i}$ and $\gamma_{i}$ such that

$$
x=\sum P_{i}\left\{\infty, \gamma_{i}(\infty)\right\}=\sum P_{i}\left\{\beta, \gamma_{i}(\beta)\right\}
$$

for any $\beta \in \mathfrak{h}^{*}$. Taking $\beta=\alpha$ proves the corollary.

\section{Remark 2.6.}

1. When $k=2$, the corollary follows from [4, Section 1], which asserts that map $\Gamma_{0}(N) \rightarrow \wp_{2}\left(\Gamma_{0}(N)\right)=H_{1}\left(X_{0}(N), \mathbb{Z}\right)$ sending $\gamma$ to $\{\alpha, \gamma(\alpha)\}$ is a surjective group homomorphism.

2. In Proposition 2.7 below, we shall prove more generally that every element of $\mathcal{M}_{k}(N, \varepsilon)$ is a sum of modular symbols of the form $P\{\alpha, \gamma(\alpha)\}$, as long as we allow $\alpha$ to vary over $\mathbb{P}^{1}(\mathbb{Q})$.

\subsection{What space do the symbols $P\{\infty, \gamma(\infty)\}$ span?}

Suppose that $N$ and $k$ are positive integers, with $k$ even.

Definition 3. For any $\alpha \in \mathbb{P}^{1}(\mathbb{Q})$, let $\mathcal{W}_{\alpha}$ denote the subspace of $\mathcal{M}_{k}\left(\Gamma_{0}(N)\right.$; $\left.\mathbb{Q}\right)$ spanned by symbols of the form $P\{\alpha, \gamma(\alpha)\}$, for $P \in V_{k-2}$ and $\gamma \in \Gamma_{0}(N)$.

Corollary 2.5 draws our attention to $\mathcal{W}_{\infty}$. Since $\mathcal{W}_{\infty}$ contains $\delta_{k}\left(\Gamma_{0}(N)\right)$, it is natural to ask how much bigger it is. As mentioned in Remark 2.6, when $k=2$, Manin proved that for any $\alpha \in \mathbb{P}^{1}(\mathbb{Q})$, we have $\mathcal{W}_{\alpha}=\mathcal{W}_{\infty}=\varsigma_{2}\left(\Gamma_{0}(N) ; \mathbb{Q}\right)$. We now compute $\mathcal{W}_{\alpha}$ for any weight $k>2$.

Proposition 2.7. Suppose that $k>2$. Then the space $W_{\alpha}$ is equal to the inverse image under the boundary map $\delta$ of the one-dimensional subspace $V_{k-2}\{\alpha\} \subset \mathscr{B}_{k}\left(\Gamma_{0}(N) ; \mathbb{Q}\right)$. Hence $\operatorname{dim} \mathcal{W}_{\alpha}=\operatorname{dim} \S_{k}\left(\Gamma_{0}(N) ; \mathbb{Q}\right)+1$ and $\mathcal{M}_{k}\left(\Gamma_{0}(N) ; \mathbb{Q}\right)=\sum_{\alpha \in \mathbb{P}^{1}(\mathbb{Q})} \mathcal{W}_{\alpha}$.

Proof. In [5, Section 1.4], Merel shows that $V_{k-2}\{\alpha\}$ has dimension 1 (see the proof of [5, Section 1.4, Proposition 4]), and that $P(X, Y)\{u / v\}$ is nonzero if $P(u, v) \neq 0$.

Corollary 2.5 implies that $\mathcal{W}_{\alpha}$ contains the kernel $\delta_{k}\left(\Gamma_{0}(N)\right)$ of the boundary map $\delta$. It thus suffices to show that $\delta\left(\mathcal{W}_{\alpha}\right)=V_{k-2}\{\alpha\}$. For $P \in V_{k-2}$ and $\gamma \in \Gamma_{0}(N)$, we have

$$
\delta(P\{\alpha, \gamma(\alpha)\})=P\{\gamma(\alpha)\}-P\{\alpha\}=\left(\gamma^{-1} P-P\right)\{\alpha\} \in V_{k-2}\{\alpha\},
$$

so $\delta\left(\mathcal{W}_{\alpha}\right) \subset V_{k-2}\{\alpha\}$. 
For $\gamma=\left(\begin{array}{cc}1 & 0 \\ N & 1\end{array}\right) \in \Gamma_{0}(N)$, we have

$$
\begin{aligned}
\delta\left(X^{k-3} Y\{\alpha, \gamma(\alpha)\}\right) & =\left(\gamma^{-1}\left(X^{k-3} Y\right)-X^{k-3} Y\right)\{\alpha\} \\
& =\left(X^{k-3}(N X+Y)-X^{k-3} Y\right)\{\alpha\} \\
& =N X^{k-2}\{\alpha\} .
\end{aligned}
$$

If $\alpha \neq 0$, then, as mentioned above, $X^{k-2}\{\alpha\} \neq 0$. (If $\alpha=0$, use $X Y^{k-3}$ and $\gamma=\left(\begin{array}{cc}1 & N \\ 0 & 1\end{array}\right)$ instead.) Because there is a nonzero element in $\delta\left(\mathcal{W}_{\alpha}\right)$ and $V_{k-2}\{\alpha\}$ has dimension 1 , it follows that $\delta\left(\mathcal{W}_{\alpha}\right)=V_{k-2}\{\alpha\}$. The final claim of the proposition is true because $\mathscr{B}_{k}\left(\Gamma_{0}(N) ; \mathbb{Q}\right)=\sum_{\alpha \in \mathbb{P}^{1}(\mathbb{Q})} V_{k-2}\{\alpha\}$.

Corollary 2.8. Fix $\alpha \in \mathbb{P}^{1}(\mathbb{Q})$. Then $\mathfrak{W}_{\alpha}=\mathcal{M}_{k}\left(\Gamma_{0}(N)\right.$; $\left.\mathbb{Q}\right)$ if and only if $N=1$.

Proof. When $N=1, \gamma$ can be any element of $\mathrm{SL}_{2}(\mathbb{Z})$, so the assertion is clear. Next, suppose that $\mathcal{W}_{\alpha}=\mathcal{M}_{k}\left(\Gamma_{0}(N) ; \mathbb{Q}\right)$. If $k=2$, then by [4, Section 1$], \mathcal{W}_{\alpha}=\wp_{k}\left(\Gamma_{0}(N) ; \mathbb{Q}\right)$, so $N=1$ since there is always a weight 2 Eisenstein series when $N>1$. Next, suppose that $k>2$. By [5, Section 1.4, Proposition 5], $\delta$ is surjective and by [5, Section 1.4, Proposition 5] the dimension of the image of $\delta$ equals $\# \Gamma_{0}(N) \backslash \mathbb{P}^{1}(\mathbb{Q})$. Combining Proposition 2.7 with our assumption that $\mathcal{W}_{\alpha}=\mathcal{M}_{k}\left(\Gamma_{0}(N) ; \mathbb{Q}\right)$ implies that $\# \Gamma_{0}(N) \backslash \mathbb{P}^{1}(\mathbb{Q})=1$, so $N=1$, as claimed.

\section{Application to computing periods of newforms}

The authors were led to introduce transportable modular symbols in order to study the error term $\left(P-\varepsilon(\gamma) \gamma^{-1} P\right)\{\infty, \alpha\}$ of equation 1 of Proposition 2.1 in the context of computing periods of newforms. There are many ways to compute periods of newforms, but we hope that the method given below will be of value in some contexts.

Section 3.1 contains an algorithm for computing periods that relies on Theorem 2.4. We present a potentially more efficient method in Section 3.2.

\subsection{An algorithm for computing periods}

Let $f=\sum a_{n} q^{n} \in S_{k}(N, \varepsilon)$ be a cuspform, and let $x \in \mathcal{M}_{k}(N, \varepsilon)$ be a modular symbol. Then $\langle f, x\rangle$ is a linear combination of integrals of the form

$$
\left\langle f, X^{m} Y^{k-2-m}\{\alpha, \infty\}\right\rangle=2 \pi i \int_{\alpha}^{i \infty} f(z) z^{m} d z,
$$

(see [5, Section 1.5]), where $\alpha \in \mathfrak{h}^{*}$ and the integer $m$ satisfies $0 \leqslant m \leqslant k-2$. If $\alpha \in \mathfrak{h}$, then the imaginary part of $\alpha$ is positive, so

$$
2 \pi i \int_{\alpha}^{i \infty} f(z) z^{m} d z=\sum_{n \geqslant 1} a_{n} c_{n},
$$

where

$$
c_{n}=2 \pi i \int_{\alpha}^{i \infty} z^{m} e^{2 \pi i n z} d z .
$$

The reversal of summation and integration is justified because the sum converges absolutely. We compute the $c_{n}$ using the following formula, which we obtain using repeated integration by parts. 


\section{Lemma 3.1.}

$$
\int_{\alpha}^{i \infty} e^{2 \pi i n z} z^{m} d z=e^{2 \pi i n \alpha} \sum_{s=0}^{m}\left\{\frac{(-1)^{s} \alpha^{m-s}}{(2 \pi i n)^{s+1}} \cdot \prod_{j=(m+1)-s}^{m} j\right\} .
$$

If $\alpha$ has large imaginary part, the $c_{n}$ will rapidly converge to 0 as $n \rightarrow \infty$. However, the reversal of summation and integration above need not be valid when $\alpha$ is a real number, so for computational purposes we are led to express periods in terms of integrals with end points that are in $\mathfrak{h}$. When $k=2$, this is easy because of the identity $\{\infty, \gamma(\infty)\}=\{\alpha, \gamma(\alpha)\}$, which is valid for any $\alpha \in \mathfrak{h}^{*}$. However, this identity can fail when $k>2$; the failure is made precise in Proposition 2.1.

Since we can take the real part of $\alpha$ to be greater than 0 , each of the terms on the right-hand side of Equation 1 can be computed using the sum given by Lemma 3.1.

We showed in Section 2 that every cuspidal modular symbol can be expressed as a sum of symbols of the form $P\{\infty, \gamma(\infty)\}$. Periods of modular symbols of this form can then be computed using the following algorithm.

Algorithm 3.2. Given a triple $\gamma \in \Gamma_{0}(N), P \in V_{k-2}$ and $g \in S_{k}(N, \varepsilon)$, this algorithm computes the period integral $\langle g, P\{\infty, \gamma(\infty)\}\rangle$.

Express $\gamma$ as $\left(\begin{array}{cc}a & b \\ c N & d\end{array}\right) \in \Gamma_{0}(N)$, and set $\alpha=(-d+i) / c N$ in Proposition 2.1.

Replacing $\gamma$ by $-\gamma$ if necessary, we find that the imaginary parts of $\alpha$ and $\gamma(\alpha)=(a+i) / c N$ are both equal to the positive number $1 / c N$.

Equation 6 and Lemma 3.1 can now be used to compute the period integrals provided by Proposition 2.1 .

\subsection{The $W_{N}$-trick}

In this section, in order to obtain a potentially more efficient way of computing periods than Algorithm 3.2, we generalize the method of Cremona [3] to even integer weight $k \geqslant 2$. In Algorithm 3.2, with $\gamma=\left(\begin{array}{cc}a & b \\ c N & d\end{array}\right)$, the endpoints of the corresponding integrals have imaginary part $1 / c N$. However, using the following trick, one can increase the imaginary part of all the endpoints involved to at least $1 / d \sqrt{N}$, which is sometimes a significant improvement.

Recall that the Atkin-Lehner involution $W=W_{N}$ is induced by the matrix $\left(\begin{array}{rr}0 & -1 \\ N & 0\end{array}\right)$; it acts on modular forms by sending a cuspform $f \in S_{k}(N, \varepsilon)$ to the form

$$
\left.f\right|_{W}(z)=N^{-k / 2} z^{-k} f(-1 /(N z)) \in S_{k}\left(N, \varepsilon^{-1}\right) .
$$

If $f$ is an eigenvector for $W$, then necessarily $\varepsilon=\varepsilon^{-1}$. For the rest of this section, we assume that $\varepsilon^{2}=1$. Then $W$ acts on $\mathcal{M}_{k}(N, \varepsilon)$ by

$$
W(P(X, Y)\{\alpha, \beta\})=\frac{P(Y,-N X)}{N^{k / 2-1}}\left\{-\frac{1}{N \alpha},-\frac{1}{N \beta}\right\},
$$

and this action is compatible with the integration pairing. 
Proposition 3.3. Let $g \in S_{k}(N, \varepsilon)$ be a cuspform that is an eigenform for the AtkinLehner involution $W$ having eigenvalue $w$. Then for any transportable modular symbol $\sum_{j=1}^{m} P_{j}\left\{\infty, \gamma_{j}(\infty)\right\}$ with $\gamma_{j} \in \Gamma_{0}(N)$ and $P_{j} \in V_{k-2}$, we have for any $\alpha \in \mathfrak{h}$ the following formula:

$$
\begin{aligned}
\left\langle g, \sum_{j=1}^{m} P_{j}\left\{\infty, \gamma_{j}(\infty)\right\}\right\rangle= & \left\langle g, \sum_{j=1}^{m} w \frac{P_{j}(Y,-N X)}{N^{k / 2-1}}\{W(\alpha), \infty\}\right. \\
& +\sum_{j=1}^{m}\left(P_{j}-w \frac{P_{j}(Y,-N X)}{N^{k / 2-1}}\right)\left\{\frac{i}{\sqrt{N}}, \infty\right\} \\
& \left.-\sum_{j=1}^{m} P_{j}\left\{\gamma_{j}(\alpha), \infty\right\}\right\rangle .
\end{aligned}
$$

Here $W(\alpha)=-1 /(N \alpha)$.

$$
\begin{aligned}
& \text { If } \gamma_{j}=\left(\begin{array}{cc}
a_{j} & b_{j} \\
c & d
\end{array}\right), \text { where } c \text { and } d \text { are fixed integers that do not depend on } j \text {, then } \\
& \qquad \begin{aligned}
\left\langle g, \sum_{j=1}^{m} P_{j}\left\{\infty, \gamma_{j}(\infty)\right\}\right\rangle= & \left\langle g, \sum_{j=1}^{m} w \frac{P_{j}(Y,-N X)}{N^{k / 2-1}}\left\{\frac{c}{d}+\frac{i}{d \sqrt{N}}, \infty\right\}\right. \\
& +\sum_{j=1}^{m}\left(P_{j}-w \frac{P_{j}(Y,-N X)}{N^{k / 2-1}}\right)\left\{\frac{i}{\sqrt{N}}, \infty\right\} \\
& \left.-\sum_{j=1}^{m} P_{j}\left\{\frac{b_{j}}{d}+\frac{i}{d \sqrt{N}}, \infty\right\}\right\rangle .
\end{aligned}
\end{aligned}
$$

Proof. By Proposition 2.1, our condition of transportability implies that we have

$$
\sum_{j=1}^{m} P_{j}\left\{\infty, \gamma_{j}(\infty)\right\}=\sum_{j=1}^{m} P_{j}\left\{\alpha, \gamma_{j}(\alpha)\right\} \text {. }
$$

The steps of the following computation are described below.

$\left\langle g, P_{j}\left\{\alpha, \gamma_{j}(\alpha)\right\}\right\rangle$

$$
\begin{aligned}
& =\left\langle g, P_{j}\left\{\alpha, \frac{i}{\sqrt{N}}\right\}+P_{j}\left\{\frac{i}{\sqrt{N}}, W(\alpha)\right\}+P_{j}\left\{W(\alpha), \gamma_{j}(\alpha)\right\}\right\rangle \\
& =\left\langle g, w \frac{W\left(P_{j}\right)}{N^{k / 2-1}}\left\{W(\alpha), \frac{i}{\sqrt{N}}\right\}+P_{j}\left\{\frac{i}{\sqrt{N}}, W(\alpha)\right\}+P_{j}\left\{W(\alpha), \gamma_{j}(\alpha)\right\}\right\rangle \\
& =\left\langle g,\left(w \frac{W\left(P_{j}\right)}{N^{k / 2-1}}-P_{j}\right)\left\{W(\alpha), \frac{i}{\sqrt{N}}\right\}+P_{j}\{W(\alpha), \infty\}-P_{j}\left\{\gamma_{j}(\alpha), \infty\right\}\right\rangle \\
& =\left\langle g, w \frac{W\left(P_{j}\right)}{N^{k / 2-1}}\{W(\alpha), \infty\}+\left(P_{j}-w \frac{W\left(P_{j}\right)}{N^{k / 2-1}}\right)\left\{\frac{i}{\sqrt{N}}, \infty\right\}-P_{j}\left\{\gamma_{j}(\alpha), \infty\right\}\right\rangle .
\end{aligned}
$$

In the first step, we break the path from $\alpha$ to $\gamma_{j}(\alpha)$ into three paths. In the second step, we apply the $W$-involution to the first term, and use the fact that the action of $W$ is compatible with the pairing $\langle$,$\rangle . The third step involves combining the first two terms, and breaking$ up the third. In the final step, we replace $\{W(\alpha), i / \sqrt{N}\}$ by $\{W(\alpha), \infty\}+\{\infty, i / \sqrt{N}\}$, and regroup. Taking the sum of both sides of the expression over $j$ from 1 to $m$ gives the first result of the proposition. 
Now, following Cremona [2, Section 2.10.8], in order to simultaneously maximize the imaginary parts of $\gamma_{j}(\alpha)$ and $W(\alpha)$, we take

$$
\alpha=\gamma_{1}^{-1}\left(\frac{b_{1}}{d}+\frac{i}{d \sqrt{N}}\right) .
$$

In this case we have

$$
W(\alpha)=\frac{c}{d}+\frac{i}{d \sqrt{N}}
$$

and

$$
\gamma_{j}(\alpha)=\frac{b_{j}}{d}+\frac{i}{d \sqrt{N}}
$$

The second formula then follows.

Remark 3.4. Let $\gamma=\left(\begin{array}{ll}a & b \\ c & d\end{array}\right) \in \Gamma_{0}(N)$. Since the imaginary parts of the terms $i / \sqrt{N}, \gamma_{j}(\alpha)$ and $W(\alpha)$ in the second part of the proposition are all relatively large, the sums appearing in Equation 6 converge relatively quickly if $d$ is small. However, we emphasize that it is extremely important to choose $\gamma_{j}$ in Proposition 3.3 with d small; otherwise, the series will converge very slowly.

\section{Examples}

The example of Section 4.1 illustrates some of the results of this paper for the weight-12 modular form $\Delta$, and Section 4.2 concerns a nonrational form of level 11 and weight 4 . The computations below were done using the first author's implementation of the algorithms of [6] in Magma [1].

\subsection{The weight-12 form $\Delta$}

Let $f=\Delta=q \cdot \prod\left(1-q^{n}\right)^{24}$ be the unique normalized eignform in $S_{12}(1)$. The space $\mathcal{M}_{12}(1 ; \mathbb{Q})$ of modular symbols has dimension 3 , and is spanned by $a_{1}=X^{10}\{0, \infty\}$, $a_{2}=X^{8} Y^{2}\{0, \infty\}$, and $a_{3}=X^{9} Y\{0, \infty\}$, and the cuspidal subspace $s_{12}(1 ; \mathbb{Q})$ has dimension 2, and is spanned by $a_{2}$ and $a_{3}$.

As explained in Example 2.2, there is a transportable modular symbol associated to each nonidentity element $\gamma \in \mathrm{SL}_{2}(\mathbb{Z})$. The transportable symbol

$$
\left(2 X^{2}+2 X Y-Y^{2}\right)^{5}\left\{\infty, \frac{1}{2}\right\}=-300 X^{9} Y\{0, \infty\}
$$

is attached to $\left(\begin{array}{ll}1 & 1 \\ 2 & 3\end{array}\right)$, and

$$
-4665600 X^{8} Y^{2}\{0, \infty\}-87300 X^{9} Y\{0, \infty\}
$$

is attached to $\left(\begin{array}{rr}-8 & 5 \\ 19 & -12\end{array}\right)$. Together, these two transportable symbols span $\varsigma_{12}(1 ; \mathbb{Q})$.

The period map $\Phi_{f}$ sends $X^{i} Y^{10-i}\{0, \infty\}$ to $2 \pi i \int_{0}^{\infty} z^{i} f(z) \mathrm{dz}$. These integrals are, up to scalars, special values of $L(f, s)$ at critical integers, so they could be computed using any of the standard methods. In any case, we obtain an approximation for the period map:

$$
\begin{aligned}
& \Phi_{f}\left(a_{1}\right) \sim 0.0374412812, \\
& \Phi_{f}\left(a_{2}\right) \sim-0.0159703242, \\
& \Phi_{f}\left(a_{3}\right) \sim-0.0232962319 i .
\end{aligned}
$$


The period lattice $\Lambda$ of $f$ is spanned by $\Phi_{f}\left((1 / 14) a_{2}\right)$ and $\Phi_{f}\left((1 / 48) a_{3}\right)$. (The fractions appear because $\wp_{12}(1 ; \mathbb{Z})$ has basis $(1 / 14) a_{2}$ and $(1 / 48) a_{3}$.) Since $\mathbb{C} / \Lambda$ is a onedimensional torus, it makes sense to consider the corresponding elliptic curve over $\mathbb{C}$. This is the elliptic curve $y^{2}=x^{3}-27 c_{4} x-54 c_{6}$, where $c_{4} \sim 28091951348793344.58$ and $c_{6} \sim-4.70682548 \times 10^{24}$. The $j$-invariant of this curve is approximately 2592849.394270 . Is $j$ a transcendental number?

\subsection{Level 11 , weight 4}

The unique normalized eigenform in $S_{4}\left(\Gamma_{0}(11)\right)$ is

$$
f=q+\alpha q^{2}+(-4 \alpha+3) q^{3}+(2 \alpha-6) q^{4}+(8 \alpha-7) q^{5}+\cdots,
$$

where $\alpha^{2}-2 \alpha-2=0$. The space $\mathcal{M}_{4}\left(\Gamma_{0}(11) ; \mathbb{Q}\right)$ has basis

$$
\begin{aligned}
& a_{1}=X^{2}\{0, \infty\}, \\
& a_{2}=\left(64 X^{2}+16 X Y+Y^{2}\right)\left\{-\frac{1}{8}, 0\right\}, \\
& a_{3}=\left(49 X^{2}+14 X Y+Y^{2}\right)\left\{-\frac{1}{7}, 0\right\}, \\
& a_{4}=\left(25 X^{2}+10 X Y+Y^{2}\right)\left\{-\frac{1}{5}, 0\right\}, \\
& a_{5}=\left(100 X^{2}+20 X Y+Y^{2}\right)\left\{-\frac{1}{10}, 0\right\}, \\
& a_{6}=Y^{2}\{\infty, 0\} .
\end{aligned}
$$

The subspace $\varsigma_{4}\left(\Gamma_{0}(11) ; \mathbb{Q}\right)$ has basis $b_{1}=a_{2}-a_{6}, b_{2}=a_{3}-a_{6}, b_{3}=a_{4}-a_{6}$, $b_{4}=a_{5}-a_{6}$.

As explained in Example 2.2, there is a transportable modular symbol associated to each nonidentity element $\gamma \in \Gamma_{0}(11)$. For example the transportable symbol

$$
\left(11 X^{2}-11 X Y+Y^{2}\right)\left\{\infty, \frac{10}{11}\right\}=11\left(a_{5}-a_{6}\right)
$$

is associated to $\gamma=\left(\begin{array}{ll}10 & -1 \\ 11 & -1\end{array}\right)$. The symbol

$$
-\frac{5}{4} b_{1}+\frac{5}{4} b_{2}-\frac{1}{4} b_{3}+\frac{1}{4} b_{4}
$$

is the transportable symbol associated to $\left(\begin{array}{r}5-1 \\ 11-2\end{array}\right)$. The symbol

$$
-\frac{9}{8} b_{1}-\frac{19}{8} b_{2}+\frac{19}{8} b_{3}+\frac{99}{8} b_{4}
$$

is associated to $\left(\begin{array}{rr}4 & 1 \\ 11 & 3\end{array}\right)$, and

$$
-\frac{27}{8} b_{1}+\frac{11}{8} b_{2}+\frac{9}{8} b_{3}+\frac{49}{8} b_{4}
$$

is associated to $\left(\begin{array}{rr}3 & -2 \\ 11 & -7\end{array}\right)$. Together, these four transportable symbols span $\varsigma_{4}\left(\Gamma_{0}(11) ; \mathbb{Q}\right)$.

In order to illustrate Section 2.3, we remark that symbols of the form $P\{\infty, \gamma(\infty)\}$ do not span all of $\mathcal{M}_{4}\left(\Gamma_{0}(11) ; \mathbb{Q}\right)$, but they do span a space bigger than $\varsigma_{4}\left(\Gamma_{0}(11) ; \mathbb{Q}\right)$. Corollary 2.5 implies that their span contains $\varsigma_{4}\left(\Gamma_{0}(11) ; \mathbb{Q}\right)$; however, the symbol $Y^{2}\{\infty, 1 / 11\}$ does not lie in $\varsigma_{4}\left(\Gamma_{0}(11) ; \mathbb{Q}\right)$.

Acknowledgements. The authors would like to thank L. Merel and J. Cremona for helpful advice, I. Kiming for improving the proof of Theorem 2.4, and the referee for several helpful remarks. 


\section{References}

1. W. Bosma, J. Cannon and C. Playoust, 'The Magma algebra system. I. The user language', J. Symbolic Comput. 24 (1997) 235-265. 179

2. J. E. CRemona, Algorithms for modular elliptic curves, 2nd edn (Cambridge University Press, Cambridge, 1997). 179

3. J. E. Cremona, 'Computing periods of cusp forms and modular elliptic curves', Experiment. Math. 6 (1997) 97-107. 177

4. J. I. Manin, Parabolic points and zeta functions of modular curves, Izv. Akad. Nauk SSSR Ser. Mat. 36 (1972) 19-66. 175, 176

5. L. Merel, Universal Fourier expansions of modular forms. On Artin's conjecture for odd 2-dimensional representations (Springer, 1994) 59-94. 170, 175, 175, 176, 176, 176

6. W. A. SteIn, 'Explicit approaches to modular abelian varieties', Ph.D. thesis, University of California, Berkeley (2000). 179

William A. Stein wasemath.harvard.edu

Department of Mathematics

Harvard University

One Oxford Street

Cambridge, MA 02138

USA

http://modular.fas.harvard.edu

Helena A. Verrill verrillemath.uni-hannover.de

Institute for Mathematics

University of Hannover

Welfengarten 1

30167 Hannover

Germany

http://hverrill.net 